PSICOLOGÍA

IBEROAMERICANA
Psicología Iberoamericana ISSN: 1405-0943

revista.psicologia@ibero.mx

Universidad Iberoamericana, Ciudad de México México

\title{
Evidencias de validez y confiabilidad de una escala para evaluar autoconcepto académico
}

Durán Fonseca, Thamir; Acle Tomasini, Guadalupe

Evidencias de validez y confiabilidad de una escala para evaluar autoconcepto académico

Psicología Iberoamericana, vol. 28, núm. 2, 2020

Universidad Iberoamericana, Ciudad de México, México

Disponible en: http://www.redalyc.org/articulo.oa?id=133964928004

Esta obra está bajo una Licencia Creative Commons Atribución 4.0 Internacional.

Esta obra está bajo una Licencia Creative Commons Atribución 4.0 Internacional.

\section{(c) (1)}

Esta obra está bajo una Licencia Creative Commons Atribución 4.0 Internacional. 


\title{
Evidencias de validez y confiabilidad de una escala para evaluar autoconcepto académico
}

Evidence of validity and reliability of a scale to evaluate academic self-concept

\author{
Thamir Durán Fonseca dandu22@hotmail.com \\ Universidad Nacional Autónoma de México (UNAM), México \\ http://orcid.org/0000-0002-3604-2662 \\ Guadalupe Acle Tomasini \\ Universidad Nacional Autónoma de México (UNAM), México \\ http://orcid.org/0000-0002-7292-3870
}

Psicología Iberoamericana, vol. 28, núm. 2, 2020

Universidad Iberoamericana, Ciudad de México, México

Recepción: 27 Abril 2020

Aprobación: 02 Septiembre 2020

Redalyc: http://www.redalyc.org/ articulo.oa?id $=133964928004$

Financiamiento

Fuente: Consejo Nacional de Ciencia y Tecnología

No de contrato: Consejo Nacional de Ciencia y Tecnología (beca 449441 y becario 299051) y el Programa de Apoyo a Proyectos de Investigación e Innovación Tecnológica (IN307419).

Beneficiario: Thamir Durán Fonseca
Resumen: El objetivo del estudio fue construir una escala para evaluar el autoconcepto académico en alumnos de primaria de la Ciudad y del Estado de México. Por ello, se organizaron dos fases con enfoque cuantitativo y diseño no experimental: (a) adaptación de la escala de Autoconcepto Académico Para Adolescentes (Ordaz-Villegas et al., 2013) y (b) obtención de evidencias de validez y confiabilidad. En la primera etapa participaron cinco psicólogas de 29 a 43 años elegidas con muestreo propositivo para valorar la calidad de los reactivos, y 264 estudiantes de cuarto a sexto grado de primaria de 8 a 12 años. En la segunda fase participaron 248 alumnos de 8 a 13 años. Todos los estudiantes fueron seleccionados con muestreo por cuotas. Los datos se analizaron con: razón e índice de validez de contenido, correlación, consistencia interna y análisis factoriales. Los resultados mostraron una escala de 12 reactivos $(\alpha=.84)$ con evidencias de validez y dos factores: autovaloración positiva $(\alpha \geq .78)$ y automonitoreo $(\alpha \geq$. 67). En conclusión, se obtuvo un instrumento adecuado para evaluar el autoconcepto académico en alumnos de primaria del valle de México. No obstante, se reconoce la falta de evidencias para utilizarla en muestras diferentes y en la obtención de puntuaciones normativas.

Palabras clave: autoconcepto académico, educación básica, escalas de medición.

Abstract: The aim of the study was to build a scale to evaluate the academic self-concept in elementary school students from Mexico City and the State of Mexico. Therefore, two stages were organized with a quantitative approach and non-experimental design: (a) adaptation of the Academic SelfConcept for Adolescents Scale (Ordaz-Villegas et al., 2013) and (b) obtaining evidence of validity and reliability. In the first phase, fivepsychologist aged between 29-years old and 43-years old, chosen through purposeful sampling participated and assessed the quality of the items. Two hundred and sixty-four students also participated in the first stage and they were from fourth to sixth grade of elementary school aged between 8-years old and 12-years old. Two hundred and sixty-four students aged between 8-years old and 13-years old participated in the second stage. All students were selected by quota sampling. The data were analyzed using content validity ratio, content validity index, correlation, internal consistency and factor analysis. The results showed a scale of 12 items $(\alpha=.84)$ with evidence of validity and two factors: positive self-assessment $(\alpha \geq .78)$ and self-monitoring $(\alpha \geq .67)$. In conclusion, an adequate scale was obtained to assess academic self-concept in elementary school students from the Valley of Mexico. However, there are limitations regarding using it in different samples and in obtaining normative scores.

Keywords: academic self-concept, basic education, measurement scales. 


\section{Introducción}

La forma en que las personas se perciben es uno de los fenómenos de estudio más antiguos en las ciencias sociales (Salum-Fares et al., 2011). Dentro de la psicología, es uno de los temas que más interés provoca (Esnaola et al., 2008; García \& Musitu, 2014; Peralta \& Sánchez, 2003), principalmente en la psicología educativa (Cazalla-Luna \& Molero, 2013; Gargallo et al., 2009; Shavelson et al., 1976). No obstante, dicha percepción ha recibido diferentes nombres, pero el autoconcepto es el constructo que más ha destacado (Cazalla-Luna \& Molero, 2013) porque permite comprender las relaciones de los individuos, sus comportamientos (García, 2003; Moreno, 2005; Shavelson et al., 1976), la personalidad (Esnaola et al., 2008) y el bienestar general (Cazalla-Luna \& Molero, 2013).

Por ello, no sorprende que existan diversos instrumentos que pretenden evaluarlo, aunque la mayoría están enfocados en adolescentes y adultos, además de que carecen de evidencias de validez de contenido. En este sentido, se considera importante construir una escala de autoconcepto académico para estudiantes de primaria, los cuales, de acuerdo con Álvarez et al. (2015) y Moreno (2005), se encuentran en una etapa significativa en la construcción de sus propias concepciones.

De acuerdo con Peralta y Sánchez (2003) no existe una definición unánime del término, pero a partir del análisis clásico de Shavelson et al. (1976) se ha considerado que el autoconcepto es la percepción que un individuo tiene de sí mismo y que se caracteriza por una serie de elementos que actualmente cuentan con evidencia empírica. Desde entonces, Shavelson et al. (1976) argumentaron que el autoconcepto es: 1) organizado-estructurado; 2) multifacético, con un sistema de categorías que incluyen áreas como la escuela o el atractivo físico; 3 ) jerárquico, con experiencias específicas en la base y un autoconcepto general en la cúspide; 4) estable en su concepción general, pero inestable en situaciones específicas; 5) evolutivo o en desarrollo; 6) evaluativo porque las personas desarrollan valoraciones sobre sí mismas; y, 7) diferenciable de otros constructos con los que está relacionado.

Sin embargo, la característica jerárquica provocó el cambio más notable en la forma de entender el autoconcepto, al reemplazar la visión unidimensional antes imperante, por una concepción multidimensional (Esnaola et al., 2008). Los planteamientos recientes reconocen de manera casi unánime (Peralta \& Sánchez, 2003), que el autoconcepto es una entidad con múltiples facetas (García \& Musitu, 2014) diferenciables entre sí (Byrne \& Shavelson, 1986). De acuerdo con Shavelson et al. (1976) el autoconcepto se divide en dos componentes, el académico y el no académico. Éste último se asocia con aspectos de la personalidad e incluye los autoconceptos emocionales, sociales y físicos (García \& Musitu, 2014; Marsh, 1990). Por su parte, el académico se vincula con el logro escolar y se estructura en áreas delimitadas por las materias escolares (Shavelson et al., 1976). Por lo tanto, el autoconcepto global puede 
interpretarse como distinto pero asociado con el autoconcepto académico (Byrne \& Shavelson, 1986).

Desde esta perspectiva, el interés por la variable tuvo implicaciones específicas en la educación, sobre todo, para comprender la conducta de los estudiantes (Salum-Fares \& Reséndiz-Balderas, 2015). En consecuencia, el estudio del autoconcepto académico tomó un camino propio y se le conceptualizó como la percepción del alumno sobre sus capacidades para realizar actividades escolares relacionadas con su aprendizaje (González, 2005; Santos \& Santos, 2013). No obstante, la investigación independiente del autoconcepto académico abrió el debate sobre su estructura en dos grandes vertientes. Por un lado, para CazallaLuna y Molero (2013) puede ser dividido en autoconceptos específicos de las materias escolares (por ejemplo, matemáticas, español, física, biología, historia, etc.), y por otro lado, autores como Esnaola et al. (2008) y Moreno (2005) argumentan sobre la existencia de un autoconcepto académico general con sólo dos dominios, matemáticas y contenidos lingüísticos.

Con base en Marsh (1990) el modelo de las materias escolares es adecuado únicamente cuando se limita a las asignaturas básicas, pero carece de sentido cuando se amplía el espectro con otras actividades académicas. Además, parte importante del apoyo en esta concepción, se basa en la decisión arbitraria sobre qué materias son esenciales. En relación, los hallazgos de Byrne y Shavelson (1986) y Marsh (1990) revelaron que el modelo de dos factores de segundo orden (matemáticas y verbal-lingüístico) se ajustó mejor a los datos, en comparación con los modelos unidimensional de Shavelson et al. (1976) y el de las asignaturas escolares. Por consiguiente, concluyeron que el autoconcepto está ordenado jerárquicamente como lo dedujeron Shavelson et al. (1976), pero la forma particular de su estructura era más complicada, porque en lugar de un solo factor, existían dos dimensiones necesarias para explicar el autoconcepto académico (Byrne \& Shavelson, 1986; Marsh, 1990).

Otra de las características más relevantes, consiste en que el desarrollo del autoconcepto se determina por la influencia de las personas significativas (Moreno, 2005; Schunk \& Pajares, 2009) y las experiencias de éxito y fracaso (García, 2003). En otras palabras, la socialización de los estudiantes con los otros, en especial la familia, los pares y los profesores, constituye la base sobre la que desarrollan las propias percepciones acerca de las habilidades y capacidades que se poseen (Shavelson et al., 1976).

Durante la primera infancia el autoconcepto es global, indiferenciado y específico de la situación (Shavelson et al., 1976), y la familia es el referente principal de su construcción (Álvarez et al., 2015), pero es en la niñez tardía cuando se integran las opiniones que los padres tienen sobre los hijos (Moreno, 2005). En cambio, la influencia de los iguales complementa el autoconcepto durante la adolescencia, y poco después se vuelve más estable (García, 2003), sus facetas se tornan más independientes -académica, emocional, social y física- (Byrne \& Shavelson, 1986) y sus auto-descripciones son más específicas, complejas 
y sutiles (Cazalla-Luna \& Molero, 2013). Según los hallazgos de Ibarra y Jacobo (2016) las comparaciones con los pares disminuyen al final de esta etapa -aunque su influjo no desaparece por completo-y experimentan fluctuaciones en los niveles del autoconcepto académico. Por ejemplo, a los 15 años sus valores decrecen en comparación con los 12, pero a los 18 aumentan y afirman tener menos dificultad para realizar las tareas escolares.

$\mathrm{Al}$ respecto, algunos estudios han respaldado la relevancia de los otros, como la influencia que los padres (Álvarez et al., 2015) y maestros ejercen en el autoconcepto académico de los alumnos (Wang \& Eccles, 2013). En representación, García (2003) encontró que los sistemas familiares que desarrollan el autoconcepto, apoyan emocionalmente, aceptan a sus individuos, ayudan en tareas y brindan expectativas sociales positivas. Por su parte, Marsh y Seaton (2013) afirman que los estudiantes que asisten a clases de alta exigencia académica tienen menos autoconcepto académico que los estudiantes igualmente capaces que están en entornos de baja o moderada exigencia.

Otros hallazgos también han mostrado la relevancia que el autoconcepto académico tiene en diversos aspectos psico-educativos. Algunos de éstos indican que los alumnos con problemas de aprendizaje presentan un autoconcepto académico más negativo en comparación con estudiantes regulares (González-Pineda et al., 2000) y que la cantidad de calificaciones reprobatorias es mayor en los alumnos con peor autoconcepto (Orgilés et al., 2012). Asimismo, éste se relaciona con el aprendizaje (Stegers-Jager et al., 2016), el rendimiento (Gargallo et al., 2009; Peralta \& Sánchez, 2003; Salum-Fares \& Reséndiz-Balderas, 2015), el logro académico (Marsh \& Seaton, 2013), las atribuciones de éxito y fracaso (Moreno, 2005), las decisiones de estudiar en la universidad (Santana et al., 2009) y la motivación escolar (Guay et al., 2010).

En parte, esta relevancia ha provocado la creación de varios instrumentos. Algunos de ellos miden el autoconcepto de manera general, a través de diferentes factores con base en el postulado de Shavelson et al. (1976), y otros sólo se enfocan en el autoconcepto académico. Las características de las escalas encontradas en la literatura se muestran en la tabla 1. 
Tabla 1

Características de los instrumentos para evaluar autoconcepto

Tabla 1

\begin{tabular}{|c|c|c|c|c|}
\hline Instrumento & $\begin{array}{c}\text { Tipo de } \\
\text { medición }\end{array}$ & Dimensiones & Población & Características psicométricas \\
\hline $\begin{array}{l}\text { Escala de autoconcepto } \\
\text { sobre las Habilidades. } \\
\text { Revisión de Merino y } \\
\text { Díaz (2003). }\end{array}$ & $\begin{array}{l}49 \text { reactivos } \\
\text { tipo Likert de } \\
5 \text { puntos. }\end{array}$ & $\begin{array}{l}7 \text { factores; matemáticas, ciencias, música, } \\
\text { lenguaje, educación física, historia y general. }\end{array}$ & $\begin{array}{c}\text { Alumnos peruanos de } \\
\text { primaria y secundaria } \\
(M=12.3 \text { años; } D E= \\
1.11) . \\
\end{array}$ & $\begin{array}{c}\text { Análisis factorial exploratorio con } 59 \% \text { de varianza } \\
\text { explicada. Análisis factorial confirmatorio: } R M S=.07 \text {; } \\
\text { índice de bondad de ajuste Tanaka }=.96 . \\
\text { Confiabilidad de } .66 \text { a } .95 \text {. }\end{array}$ \\
\hline $\begin{array}{l}\text { Escala de Autoconcepto } \\
\text { Académico (Schmidt et } \\
\text { al., 2008). }\end{array}$ & $\begin{array}{l}20 \text { reactivos } \\
\text { tipo Likert de } \\
5 \text { puntos. }\end{array}$ & 2 factores; rendimiento y autoeficacia. & $\begin{array}{l}\text { Alumnos argentinos } \\
\text { de bachillerato }(M= \\
16 \text { años; } D E=1.5) \text {. }\end{array}$ & $\begin{array}{c}\text { Validez de criterio } r=.47, p<.01 \text { con actitud hacia la } \\
\text { escuela y el estudio, y diferencias entre grupos con } \\
\text { base en el rendimiento }(F(2,580)=49.73, p<.05) \text {. } \\
\text { Análisis factorial exploratorio con } 43 \% \text { de varianza } \\
\text { explicada. Confiabilidad de } .68 \text { y } .75 \text {. }\end{array}$ \\
\hline $\begin{array}{l}\text { Escala de Autoconcepto } \\
\text { Académico Para } \\
\text { Adolescentes (Ordaz- } \\
\text { Villegas et al., 2013). }\end{array}$ & $\begin{array}{l}16 \text { reactivos } \\
\text { tipo Likert de } \\
5 \text { puntos. }\end{array}$ & $\begin{array}{l}4 \text { factores; autorregulación, aptitudes } \\
\text { intelectuales generales, motivación y } \\
\text { creatividad. }\end{array}$ & $\begin{array}{c}\text { Alumnos mexicanos } \\
\text { de bachillerato }(M= \\
16.17 \text { años; } D E= \\
1.08) \text {. }\end{array}$ & $\begin{array}{c}\text { Análisis factorial exploratorio con } 44.72 \% \text { de varianza } \\
\text { explicada. Confiabilidad de } .82 \text {. }\end{array}$ \\
\hline $\begin{array}{l}\text { Prueba de } \\
\text { Autoconcepto } \\
\text { Académico (Chávez, } \\
\text { 2014). }\end{array}$ & $\begin{array}{l}31 \text { reactivos } \\
\text { tipo Likert de } \\
7 \text { puntos }\end{array}$ & $\begin{array}{l}6 \text { factores; matemáticas, español, educación } \\
\text { física, historia, ciencias naturales y educación } \\
\text { artísticas. }\end{array}$ & $\begin{array}{c}\text { Alumnos mexicanos } \\
\text { de primaria }(M=11.3 \\
\text { años; } D E=.85) .\end{array}$ & $\begin{array}{l}\text { Análisis factorial exploratorio con } 64 \% \text { de varianza } \\
\text { explicada. Confiabilidad de } .85 \text {. }\end{array}$ \\
\hline $\begin{array}{l}\text { Autoconcepto Forma } 5 . \\
\text { Versión de Bustos et al., } \\
\text { (2015). }\end{array}$ & $\begin{array}{l}30 \text { reactivos } \\
\text { tipo Likert de } \\
11 \text { puntos. }\end{array}$ & $\begin{array}{l}5 \text { factores; académico, social, emocional, } \\
\text { familiar y fisico. }\end{array}$ & $\begin{array}{c}\text { Estudiantes } \\
\text { universitarios } \\
\text { peruanos (Mediana = } \\
19.5 \text { años). }\end{array}$ & $\begin{array}{c}\text { Validez de criterio } r>.19, p<.01 \text { con esperanza y } \\
\text { satisfacción con la vida. Análisis factorial } \\
\text { confirmatorio: } X^{2}(395)=1484.46, p<.01 ; C F I=.93 ; \\
B B N F I=.92 ; I F I=.93 ; R M S E A=.07(I C 90 \%=.07- \\
.08) \text {. Confiabilidad de } .73 \text { a } .82 \text {. El factor académico } \\
\text { posee } 6 \text { items y alfa de } .81 \text {. }\end{array}$ \\
\hline $\begin{array}{l}\text { Autoconcepto } \\
\text { Dimensional } \\
\text { (Fernández-Zabala et } \\
\text { al., 2015). }\end{array}$ & $\begin{array}{l}33 \text { reactivos } \\
\text { tipo Likert de } \\
5 \text { puntos. }\end{array}$ & $\begin{array}{l}11 \text { factores; académico verbal, académico } \\
\text { matemático, habilidad y condición física, } \\
\text { atractivo físico, fuerza física, honradez, ajuste } \\
\text { emocional, autonomia, autorrealización, } \\
\text { responsabilidad social y competencia social. }\end{array}$ & $\begin{array}{l}\text { Estudiantes } \\
\text { universitarios } \\
\text { españoles }(M=20.51 \\
\text { años; } D E=3.82) \text {. }\end{array}$ & $\begin{array}{c}\text { Valide de criterio } r=.59, p<.001 \text { con satisfacción con } \\
\text { la vida. Análisis factorial exploratorio con } 75.51 \% \text { de } \\
\text { varianza explicada. Alfa de Cronbach de } .77 \text { y omega } \\
\text { de McDonald de. } 97 \text {. Los factores académicos se } \\
\text { componen de } 4 \text { ítems cada uno. }\end{array}$ \\
\hline $\begin{array}{l}\text { Escala de Autoconcepto } \\
\text { AF5. Revisión de } \\
\text { Carranza y Bermúdez- } \\
\text { Jaimes (2017). }\end{array}$ & $\begin{array}{l}25 \text { reactivos } \\
\text { tipo Likert. }\end{array}$ & $\begin{array}{l}5 \text { factores; académico, social, emocional, } \\
\text { familiar y fisico. }\end{array}$ & $\begin{array}{l}\text { Estudiantes } \\
\text { universitarios } \\
\text { peruanos }(M=20 \\
\text { años; } D E=2.6) \text {. }\end{array}$ & $\begin{array}{c}\text { Análisis factorial exploratorio con } 51.98 \% \text { de varianza } \\
\text { explicada. Análisis factorial confirmatorio: } X^{2}=869.67 ; \\
g I=247 ; p<.001, T L I=.90 ; C F I=.92 ; G F I=.92 ; \\
A G F I=.90 \text { y } R M S E A=.05 \text {. Confiabilidad de } .77 \text { a } .83 \text {. } \\
\text { El factor académico posee } 6 \text { ítems y alfa de } .83 \text {. }\end{array}$ \\
\hline $\begin{array}{l}\text { Escala de Autoconcepto } \\
\text { Académico (Gálvez- } \\
\text { Nieto et al., 2017). }\end{array}$ & $\begin{array}{l}13 \text { reactivos } \\
\text { tipo Likert de } \\
5 \text { puntos. }\end{array}$ & $\begin{array}{l}2 \text { factores; rendimiento académico y } \\
\text { autoeficacia académica. }\end{array}$ & $\begin{array}{l}\text { Alumnos chilenos de } \\
\text { bachillerato }(M=16.3 \\
\text { años; } D E=1.37) .\end{array}$ & $\begin{array}{c}\text { Análisis factorial exploratorio con } 47.41 \% \text { de varianza } \\
\text { explicada. Análisis factorial confirmatorio: } S B-x 2(g /= \\
64)=153.93, p<.001 ; C F I=.96 ; T L I=.95 ; R M S E A= \\
.061(\text { IC } 90 \%=.049-.073) \text {. Confiabilidad de } .74 \text { y } \\
.78 .\end{array}$ \\
\hline
\end{tabular}

En la tabla 1 se puede observar que existen divergencias sobre la cantidad de reactivos y factores en las escalas. En algunos casos, el instrumento es unidimensional y en otros existen siete dimensiones, por lo que resulta obvio encontrar escalas con seis y 49 reactivos. Otra de las divergencias más notables es la población, desde alumnos de primaria hasta universitarios de cinco países distintos; Perú, Argentina, México, España y Chile. No obstante, la cantidad de opciones de respuesta es más homogénea, en donde la mayoría de los instrumentos cuentan con cinco puntos. Respecto con sus características psicométricas, en todos los casos se muestran evidencias de confiabilidad y validez, pero sólo en cuatro de las escalas se incluyó información sobre la validez de criterio y en ninguna de ellas se mostraron datos respecto a la validez de contenido.

De acuerdo con la American Educational Research Association, la American Psychological Association y el National Council on Measurement in Education (2014), la validez es el principal aspecto en las pruebas, y los datos psicométricos mostrados en los instrumentos de la tabla 1 aportan información relevante sobre diferentes aspectos de ésta, pero son insuficientes para la validación de pruebas, ya que se requieren evidencias de contenido, criterio y constructo dentro de la concepción de validez como concepto general.

En este sentido, la escala de Autoconcepto Académico Para Adolescentes (AAPA) de Ordaz-Villegas et al. (2013) se considera adecuada para la construcción de otros instrumentos, para medir el autoconcepto académico en alumnos mexicanos. Las razones más significativas son: a) se realizó con adolescentes mexicanos; b) posee pocos 
reactivos; c) cuenta con un porcentaje de varianza explicada significativo; d) tiene una confiabilidad satisfactoria; y, e) es la única escala con una concepción global del autoconcepto y con reactivos de las dimensiones de matemáticas y verbal-lingüística, en lugar de una perspectiva dividida por asignaturas escolares.

Para Ordaz-Villegas et al. (2013) la especificidad por materias ha causado problemas asociados con la evaluación, especialmente porque se excluyen algunas actividades importantes y se pierden las posibilidades de descubrir dimensiones potencialmente compensadas. Para las autoras, los estudiantes suelen tener valores diferentes en las asignaturas académicas, pero cuando las puntuaciones se agrupan, se produce un efecto de compensación entre ellas.

Desde esta perspectiva, se considera importante construir una escala de autoconcepto académico para estudiantes de primaria, porque en esta etapa se sientan las bases del aprendizaje, a través de la lectura, la comprensión de textos y la creación de escritos (Montealegre \& Forero, 2006). También, se adquieren y desarrollan aspectos adaptativos hacia la vida académica que se conservan a lo largo de la educación (Jackson, 1991) como las preferencias por algunas actividades escolares (Macionis \& Plumer, 2007). Por ello, el objetivo del estudio fue construir una escala para evaluar el autoconcepto académico en alumnos de primaria. No obstante, para lograr dicho propósito y debido a la necesidad de múltiples fuentes de evidencias en el proceso de validación (American Educational Research Association, American Psychological Association \& National Council on Measurement in Education, 2014), se optó por realizar un estudio con dos fases en muestras diferentes. En la primera se adaptó la escala AAPA de Ordaz-Villegas et al. (2013) y en la segunda se evaluaron las propiedades psicométricas de la escala construida.

\section{Método Fase 1}

\section{Características del Estudio}

La investigación se basó en el enfoque cuantitativo con diseño no experimental de tipo transversal. Para Creswell (2015) los estudios cuantitativos se caracterizan en describir tendencias por medio de la evaluación con datos numéricos en una población numerosa. Por su parte, en los diseños no experimentales de tipo transversal, se carece de manipulación de variables y asignación aleatoria, por lo que se realiza en un periodo corto de tiempo, en los contextos naturales de los participantes y después de que ocurrió el fenómeno (Kerlinger \& Lee, 2002).

\section{Objetivo}

Adaptar la escala de Autoconcepto Académico Para Adolescentes (AAPA) de Ordaz-Villegas et al. (2013) orientándola para alumnos de $4^{\circ}$ a $6^{\circ}$ grado de primaria de 8 a 12 años de edad. 


\section{Participantes}

Participaron cinco psicólogas de 29 a 43 años como jueces expertos $(M=35$ años, $D E=5)$ para evaluar la calidad de los reactivos. Éstas fueron elegidas a través de un muestreo propositivo, que consistió en una selección intencional de la muestra con base en su experiencia en psicometría $(M=3.4$ años, $D E=1.2)$ y en la evaluación psicoeducativa de estudiantes de primaria $(M=6$ años, $D E=2.3)$. Todas formaban parte del posgrado en psicología, tres como alumnas y dos como docentes. El $80 \%$ contaba con estudios de maestría y el $20 \%$ de licenciatura.

En el piloteo de la escala participaron 14 estudiantes de 8 a 12 años $(M$ $=10.21 ; D E=1.36$ ), nueve niños y cinco niñas. Cinco de los alumnos estudiaban en cuarto, cuatro en quinto y cinco en sexto grado. Éstos fueron seleccionados por medio de un muestreo accidental con base en su disponibilidad y acceso en una comunidad del Estado de México. Además de los 14 estudiantes del piloteo, para la aplicación de la escala participaron 250 alumnos de cuarto a sexto grado de primarias públicas de la zona oriente de la Ciudad de México, seleccionados a través del muestreo no probabilístico por cuotas. El 29.6\% eran de cuarto, 37.2\% de quinto y $33.2 \%$ de sexto. Su edad osciló entre los 8 y los 12 años $(M=$ 9.91; $D E=.89)$. El $53.2 \%$ fueron niñas y el $46.8 \%$ fueron niños.

\section{Instrumentos}

La adaptación de la prueba se basó en la escala de Autoconcepto Académico Para Adolescentes (AAPA) de Ordaz-Villegas et al. (2013). Las autoras desarrollaron 28 reactivos con cinco opciones de respuesta que van de nunca a siempre y cuatro factores: autorregulación; aptitudes intelectuales generales; motivación; $y$, creatividad. La escala explica el $44.72 \%$ de la varianza, con un alfa de Cronbach global de .82 y fue elaborada con estudiantes de la Ciudad de México de 14 a 18 años.

Se utilizó la planilla sobre validación de contenido de Escobar-Pérez y Cuervo-Martínez, (2008) para obtener evidencias de este tipo de validez. Consiste en un formato en el que los jueces emiten sus calificaciones respecto a la suficiencia, claridad, coherencia y relevancia de los reactivos de una escala. Contiene cuatro opciones de evaluación: 1) No cumple con el criterio; 2) Bajo nivel; 3) Moderado nivel; y, 4) Alto nivel.

Para obtener evidencias de validez de criterio se utilizó la Escala de Motivación Escolar para Alumnos de Primaria (Durán \& Acle, 2019). Ésta se compone de 16 reactivos, cinco opciones de respuesta pictográficas $\mathrm{y}$ tres factores; persistencia $(\alpha=.81)$, esfuerzo $(\alpha=.79)$ y disposición por aprobar exámenes $(\alpha=.71)$. Mostró un alfa de Cronbach de .88 con la muestra del presente estudio y posee evidencias de validez de contenido, criterio y constructo. 


\section{Procedimiento}

Se modificaron los reactivos de la escala AAPA (Ordaz-Villegas et al., 2013) a las características de la muestra. La mayoría de los ítems se cambiaron al lenguaje y contexto escolar de los estudiantes de primaria, uno de ellos se incluyó sin cambios y otros fueron eliminados por considerar que no pertenecían al autoconcepto académico, puesto que el autoconcepto se relaciona con otras variables, pero es diferenciable de éstas (Shavelson et al., 1976). La adaptación se describe con precisión en los resultados. Después, se realizó la validación con cinco colegas del posgrado en psicología de la Universidad Nacional Autónoma de México (UNAM), quienes evaluaron la calidad de los reactivos a través de la planilla sobre validación de contenido (Escobar-Pérez \& Cuervo-Martínez, 2008). Con base en esta evaluación, se construyó la versión piloto. Ésta fue aplicada de manera individual a 14 niños de una comunidad del Estado de México. En este caso, los niños fueron convocados por medio de anuncios colocados en una escuela primaria. La información recabada en el piloteo indicó que todos los reactivos eran adecuados, por lo cual, no se realizaron modificaciones.

Posteriormente, se aplicaron la versión adaptada de la AAPA y la Escala de Motivación Escolar para Alumnos de Primaria (Durán \& Acle, 2019) a 250 estudiantes provenientes de tres escuelas ubicadas al oriente de la Ciudad de México. El acceso a las primarias fue facilitado por las psicólogas del jueceo que evaluaron los ítems, las cuales han trabajado en dichos centros educativos desde hace más de tres años. Las aplicaciones de los instrumentos fueron grupales, en los salones de clase y en horario escolar convenido con los maestros. Finalmente, se averiguó el promedio de calificaciones del último trimestre de éstos alumnos.

Cabe destacar que la participación de todos los niños fue voluntaria y aprobada por las autoridades académicas de las escuelas. Además, se obtuvieron los consentimientos informados de sus padres. Para ello, se les mandaron formularios a través de los alumnos, con el propósito de que autorizaran la participación de sus hijos mediante su firma. En dicho formulario se indicó el propósito del estudio, la institución educativa involucrada, un correo electrónico de contacto para resolver dudas y las cuestiones éticas de participación voluntaria, anónima y confidencial.

\section{Análisis de Datos}

Las evaluaciones de los jueces fueron analizadas a través de la Razón de Validez de Contenido (RVC) e Índice de Validez de Contenido (IVC) propuestos por Tristán-López (2008). Asimismo, se utilizó la prueba de Kolmogorov-Smirnov para decidir el tipo de análisis adecuado en la validez de criterio. Con base en los resultados sobre la violación del supuesto de distribución normal en las puntuaciones globales de la Escala de Motivación Escolar para Alumnos de Primaria y en el promedio de calificaciones de los niños, se decidió calcular correlaciones de Spearman con dichas variables para obtener evidencias de validez de criterio. 
En la validez de constructo se realizó un Análisis Factorial Exploratorio (AFE). El tratamiento estadístico propuesto por Reyes-Lagunes et al. (2008) fue utilizado para la discriminación de los reactivos por distribución de frecuencias, sesgo y diferencias en grupos extremos. Sin embargo, se tomaron en cuenta otras consideraciones durante el cálculo del AFE. Con base en Lloret-Segura et al. (2014) no existe una procedimiento universal para ejecutar dicha técnica, porque las decisiones adecuadas dependen de las condiciones específicas de cada investigación.

En el caso particular del presente estudio, se decidió utilizar una extracción de máxima verosimilitud con matriz de correlaciones de Pearson y una rotación oblimin con base en los siguientes criterios propuestos por Lloret-Segura et al. (2014): a) se tiene que comprobar la adecuación de los datos mediante la prueba $K M O$; b) es adecuado utilizar la matriz de correlaciones de Pearson cuando existen mínimo cinco opciones de respuesta, las distribuciones de los reactivos son aproximadamente normales (asimetría y curtosis en el rango de - 1 a 1) y la muestra se compone entre 200 y 300 participantes. Además, otro criterio para Ferrando y Lorenzo-Seva (2014) consiste en que las correlaciones inter-ítem deben tener magnitudes de .50 o menos; c) el método de máxima verisimilitud es apropiado para la estimación de los factores si los ítems tienen distribuciones normales (asimetría y curtosis menores que 2 ), incluso cuando los reactivos son ordinales; d) el número de factores a retener depende de la cantidad de ítems (mínimo 4), el tamaño de la saturación (mínimo .40) y la interpretabilidad de la solución con base en la teoría; y, e) se recomienda la rotación oblicua aunque los factores parezcan independientes, entre los destaca oblimin directo.

Finalmente, se calculó el alfa de Cronbach para obtener la confiabilidad. Todos los análisis fueron ejecutados por medio del programa SPSS (v. 24).

\section{Resultados Fase 1}

\section{Adaptación de la Escala y Validez de Contenido}

Durante el proceso de adaptación de la AAPA (Ordaz-Villegas et al., 2013) se descartaron siete reactivos, se adecuaron 20 y uno se consideró apropiado para incluirlo sin cambios, por lo que finalmente se obtuvieron 21 ítems. Las modificaciones realizadas se muestran en la tabla 2. 
Tabla 2

Modificaciones realizadas en los reactivos de la escala AAPA

Tabla 2

Modificaciones realizadas en los reactivos de la escala AAPA

\begin{tabular}{|c|c|c|}
\hline Reactivos de la escala AAPA & Decisión & Adaptación \\
\hline $\begin{array}{l}\text { 1. Antes de empezar una tarea analizo las distintas formas de } \\
\text { levarlo a cabo. }\end{array}$ & Modificación & 1. Soy capaz de realizar mi tarea de distintas formas. \\
\hline $\begin{array}{l}\text { 2. Puedo repetir exactamente alguna historia que me hayan } \\
\text { contado. }\end{array}$ & Modificación & $\begin{array}{l}\text { 2. Cuando me cuentan una historia en clase puedo repetirla con } \\
\text { exactitud. }\end{array}$ \\
\hline $\begin{array}{l}\text { 3. Cuando quiero mejorar mi salud o apariencia física tomo } \\
\text { decisiones y las cumplo hasta lograr la meta. }\end{array}$ & Eliminación del reactivo & \\
\hline 4. Cumplo las fechas en las que planeo terminar mis tareas. & Modificación & $\begin{array}{l}\text { 3. Puedo entregar mis tareas en la fecha en que me dicen mis } \\
\text { maestros. }\end{array}$ \\
\hline 5. Expreso verbalmente mis ideas de una forma clara. & Modificación & 4. Soy bueno para decir mis ideas de forma en que se entiendan. \\
\hline $\begin{array}{l}\text { 6. Las actividades que me gustan (cantar, bailar, tocar un } \\
\text { instrumento) las practico mucho para hacerlo muy bien. }\end{array}$ & Modificación & $\begin{array}{l}\text { 5. Las actividades artísticas (dibujar, bailar y cantar) que me } \\
\text { dejan en la escuela las puedo hacer muy bien. }\end{array}$ \\
\hline 7. Leo varias veces los textos para encontrar la idea principal. & Modificación & 6. Puedo encontrar la idea principal de los textos que leo. \\
\hline $\begin{array}{l}\text { 8. Cuando tengo un problema busco nuevas estrategias para } \\
\text { solucionarlo. }\end{array}$ & Modificación & $\begin{array}{l}\text { 7. Soy capaz de encontrar nuevas formas de resolver un problema } \\
\text { matemático. }\end{array}$ \\
\hline 9. Puedo transformar los conceptos nuevos a mi propio lenguaje. & Modificación & $\begin{array}{l}\text { 8. Los temas que aprendo puedo explicarlos en mis propias } \\
\text { palabras. }\end{array}$ \\
\hline 10. Se me dificulta memorizar. & Modificación & 9. Soy malo para memorizar los temas que me enseñan en clase. \\
\hline $\begin{array}{l}\text { 11. Me gusta encontrar el origen de un problema para darle la } \\
\text { mejor solución. }\end{array}$ & Eliminación del reactivo & \\
\hline $\begin{array}{l}\text { 12. Analizo mis calificaciones para ver si corresponden a lo que } \\
\text { hice. }\end{array}$ & Modificación & 10. Puedo obtener buenas calificaciones. \\
\hline $\begin{array}{l}\text { 13. Me esfuerzo para que mis trabajos sean los mejores de la } \\
\text { clase. }\end{array}$ & Modificación & 11. Soy capaz de hacer mis trabajos mejor que mis compañeros. \\
\hline 14. Requiero pocas instrucciones para poder hacer una tarea. & Modificación & $\begin{array}{l}\text { 12. Soy bueno para entender las instrucciones que me dicen en } \\
\text { clase. }\end{array}$ \\
\hline 15. Puedo prever las consecuencias de un hecho o evento. & Eliminación del reactivo & \\
\hline 16. Busco más información para complementar mis materias. & Modificación & $\begin{array}{l}\text { 13. Cuando hago mis tareas puedo encontrar información que me } \\
\text { ayuda a entender mejor. }\end{array}$ \\
\hline 17. Me gusta realizar mis tareas usando mis ideas y mis formas. & Modificación & 14. Soy hábil para pensar ideas de cómo hacer mis trabajos. \\
\hline 18. Tengo más de una forma para resolver un problema. & Eliminación del reactivo & \\
\hline 19. Examino el temario antes de iniciar el semestre. & Eliminación del reactivo & \\
\hline 20. Puedo hacer cuentas mentalmente de forma fácil. & $\begin{array}{l}\text { Reactivo adecuado. Se aceptó } \\
\text { sin modificaciones. }\end{array}$ & 15. Puedo hacer cuentas mentalmente de forma fácil \\
\hline $\begin{array}{l}\text { 21. Cuando participo en un juego por equipos, hago todo para } \\
\text { que ganemos. }\end{array}$ & Modificación & 16. Soy capaz de trabajar en equipo. \\
\hline $\begin{array}{l}\text { 22. Me gustan las situaciones donde puedo comparar e } \\
\text { identificar diferentes puntos de vista o ideas en conflicto. }\end{array}$ & Modificación & 17. Puedo clasificar diferente información sobre un mismo tema. \\
\hline 23. Me gusta aprender explorando libremente. & Modificación & 18. Soy capaz de aprender al buscar información por mí mismo. \\
\hline $\begin{array}{l}\text { 24. Antes de iniciar una tarea o proyecto lo reviso para saber qué } \\
\text { estrategia voy a ocupar. }\end{array}$ & Modificación & 19. Puedo planear como voy a realizar una tarea. \\
\hline 25. Puedo crear nuevas ideas sin conocer a fondo el tema. & Modificación & $\begin{array}{l}\text { 20. Cuando escucho el título de un tema soy capaz de } \\
\text { imaginarme lo que voy a aprender }\end{array}$ \\
\hline $\begin{array}{l}\text { 26. Para solucionar un problema busco maneras que a otros no } \\
\text { se les ocurre. }\end{array}$ & Eliminación del reactivo & \\
\hline $\begin{array}{l}\text { 27. Llego puntual a la escuela porque calculo el tiempo que } \\
\text { hago, teniendo en cuenta el tráfico de la ciudad. }\end{array}$ & Eliminación del reactivo & \\
\hline $\begin{array}{l}\text { 28. Cuando tengo que exponer en clase practico previamente en } \\
\text { casa. }\end{array}$ & Modificación & 21. Soy bueno para preparar los temas que voy a exponer. \\
\hline
\end{tabular}

Cabe destacar que las modificaciones en los reactivos se realizaron con base en la complejidad de las afirmaciones para el lenguaje de los niños y en la definición del autoconcepto académico descrita en el presente estudio. Por ello, se cambiaron algunos aspectos en los ítems de la prueba de Ordaz-Villegas et al. (2013) con el propósito de que fueran comprensibles para los alumnos de $4^{\circ}$ a $6^{\circ}$ grado de primaria y que correspondieran únicamente con el constructo a evaluar. Otros reactivos fueron eliminados porque no pertenecían a la variable, por ejemplo, los ítems 11,18 y 26 correspondían con la creatividad y el tercero se refería al autoconcepto físico y no académico. 
Estos cambios influyeron en la validez de contenido, en términos de la claridad de los ítems para los niños y en la relación lógica de los reactivos con el autoconcepto académico. De acuerdo con la puntuación otorgada por los jueces, los reactivos fueron suficientes para medir la variable. La media en esta área fue de 4, la cual corresponde con la opción de "Alto nivel”. Debido a la unanimidad en los expertos, la proporción de acuerdo fue perfecta $(\mathrm{RVC}=1 ; \mathrm{IVC}=1)$. Respecto con la claridad, coherencia y relevancia, en todos los reactivos se obtuvieron valores iguales o superiores a 3.8 ("Alto nivel”), RVC superiores a .58, y el IVC fue de .88. Con base en los datos y Tristán-López (2008) la escala mostró evidencia de validez de contenido.

\section{Validez de Constructo, Criterio y Confiabilidad}

Se verificó el cumplimiento de los criterios propuestos por Lloret-Segura et al. (2014) para realizar un análisis factorial exploratorio con extracción de máxima verosimilitud, matriz de correlaciones de Pearson y rotación oblimin. Las características del presente estudio congruentes con tales supuestos fueron las siguientes: muestra de 250 participantes; cinco alternativas de respuesta en los reactivos; ítems con niveles de asimetría y curtosis en torno a 1 ; y, correlaciones inter-ítem entre .12 y .48. Por otra parte, con base en la discriminación de reactivos por distribución de frecuencias, se decidió eliminar dos ítems por tener porcentajes iguales o superiores al 50\% en la quinta opción de respuesta (siempre). Éstos fueron; 3. Puedo entregar mis tareas en la fecha en que me dicen mis maestros y 5. Las actividades artísticas (dibujar, bailar y cantar) que me dejan en la escuela las puedo hacer muy bien. En la discriminación por sesgo (asimetría de .01 a 1.14 y curtosis de .19 a .82) y por diferencias en grupos extremos $(t \geq 6.75 ; p<.001)$ los reactivos mostraron ser apropiados.

Posteriormente, el análisis factorial exploratorio reveló una estructura de dos factores que explicaron el $38.46 \%$ de la varianza. El primer factor aportó $31.36 \%$ con ocho reactivos, que correspondieron con la capacidad positiva y auto-percibida para realizar las actividades escolares. El segundo factor se constituyó de cuatro ítems y aportó el 7.1\% de la explicación de la varianza. Este último agrupó reactivos sobre la capacidad de automonitoreo en el empleo de procesos cognitivos necesarios para ejecutar actividades académicas. El valor de $K M O$ fue .90 y el ajuste mostró valores de $\chi^{2}[(134)=205.63, p<.001]$, los cuales se consideran satisfactorios de acuerdo con Lloret-Segura et al. (2014). Los resultados del análisis se muestran en la tabla 3. 
Tabla 3

Escala de Autoconcepto Académico para Alumnos de Primaria: Cargas factoriales e índices psicométricos

Tabla 3

Escala de Autoconcepto Académico para Alumnos de Primaria: Cargas factoriales e índices psicométricos

\begin{tabular}{|c|c|c|}
\hline \multirow[b]{2}{*}{ Reactivos } & Factor 1 & Factor 2 \\
\hline & $\begin{array}{l}\text { Autovaloración } \\
\text { positiva }\end{array}$ & Automonitoreo \\
\hline 10. Puedo obtener buenas calificaciones & .65 & \\
\hline 14. Soy hábil para pensar ideas de cómo hacer mis trabajos & .65 & \\
\hline $\begin{array}{l}\text { 12. Soy bueno para entender las instrucciones que me dicen en } \\
\text { clase }\end{array}$ & .64 & \\
\hline 21. Soy bueno para preparar los temas que voy a exponer & .63 & \\
\hline 11. Soy capaz de hacer mis trabajos mejor que mis compañeros & .58 & \\
\hline $\begin{array}{l}\text { 7. Soy capaz de encontrar nuevas formas de resolver un problema } \\
\text { matemático }\end{array}$ & .54 & \\
\hline 15. Puedo hacer cuentas mentalmente de forma fácil & .49 & \\
\hline 4. Soy bueno para decir mis ideas de forma en que se entiendan & .48 & \\
\hline 17. Puedo clasificar diferente información sobre un mismo tema & & .67 \\
\hline $\begin{array}{l}\text { 2. Cuando me cuentan una historia en clase puedo repetirla con } \\
\text { exactitud }\end{array}$ & & .65 \\
\hline 19. Puedo planear como voy a realizar una tarea & & .46 \\
\hline 6. Puedo encontrar la idea principal de los textos que leo & & .42 \\
\hline Total & & \\
\hline Número de reactivos & 8 & 4 \\
\hline \% de varianza explicada & 31.36 & 7.1 \\
\hline
\end{tabular}

A partir de los datos obtenidos en el análisis factorial exploratorio, se decidió descartar siete reactivos por tener aportes menores a .40 , o por aportar de manera significativa en ambos factores. Los ítems eliminados fueron; $1,8,9,13,16,18$ y 20 . Con base en la categorización de los reactivos, el autoconcepto académico se refiere a la percepción sobre la capacidad para realizar actividades escolares (autovaloración positiva) y a la percepción sobre la capacidad para ejecutar procesos cognitivos (auto-monitoreo). En cuanto a la validez de criterio, los resultados en la prueba de Kolmogorov-Smirnov mostraron que existían diferencias significativas entre la distribución normal y las distribuciones de los datos de la motivación escolar $(p=.001)$ y las calificaciones $(p<.001)$. En consecuencia, se decidió realizar correlaciones de Spearman, las cuales 
indicaron relaciones lineales y positivas con la motivación escolar $(r=.67$, $p<.01)$ y las calificaciones de los niños $(r=.23, p<.01)$.

Finalmente, el Alfa de Cronbach global reveló un valor de .84 y todos los reactivos correlacionaron de manera significativa con el puntaje total $(r \geq .23, p \leq .05)$. A mbos factores correlacionaron de manera significativa $(r=.59 ; p<.001)$ y el primero de ellos obtuvo una consistencia interna de .80 y el segundo de .67. Puesto que el coeficiente de fiabilidad depende de la cantidad de reactivos en la escala (Martínez et al., 2014), con base en Argibay (2006) se considera que los valores menores a .70 son buenos indicadores en instrumentos con longitudes reducidas. Por lo tanto, los datos obtenidos en las correlaciones y en las alfas de Cronbach mostraron evidencias adecuadas de validez de criterio y confiabilidad.

\section{Método Fase 2}

De acuerdo con criterios recientes en psicometría, resulta erróneo considerar que la validación es una característica del instrumento, por ello, es equivocado suponer que existen pruebas válidas. En cambio, actualmente se concibe a ésta como el grado en que la evidencia acumulada respalda las interpretaciones adecuadas de las puntaciones obtenidas. Es decir, lo que se evalúa son las interpretaciones de los datos y no los instrumentos de medición. En consecuencia, la validación implica acumular múltiples fuentes de evidencia científica (American Educational Research Association, American Psychological Association \& National Council on Measurement in Education, 2014). Es por ello que se consideró adecuada una segunda fase para evaluar las propiedades psicométricas de la Escala de Autoconcepto Académico para Alumnos de Primaria (EAAAP) en una muestra diferente, con base en la tendencia actual del uso secuencial de análisis factorial exploratorio y confirmatorio expuesta por Lloret-Segura et al. (2014).

\section{Objetivo}

Evaluar las propiedades psicométricas de la Escala de Autoconcepto Académico para Alumnos de Primaria (EAAAP).

\section{Participantes}

La muestra fue elegida con muestreo no probabilístico por cuotas y se conformó de 248 alumnos de cuarto a sexto grado, pertenecientes a cuatro primarias públicas de la Zona Metropolitana del Valle de México. El $34.7 \%$ eran de cuarto, $29 \%$ de quinto y $36.3 \%$ de sexto. Su edad osciló entre los 8 y los 13 años $(M=10.16 ; D E=.96)$, el $56.9 \%$ fueron niñas y el $43.1 \%$ fueron niños. 


\section{Instrumento}

Escala de Autoconcepto Académico para Alumnos de Primaria (EAAAP), elaborada en la primera fase del presente estudio. Se compone de dos secciones. En la primera se muestran la presentación, la solicitud de datos sociodemográficos, las instrucciones, ejemplos de llenado y las aclaraciones éticas. En la segunda se incluyen 12 reactivos tipo Likert con cinco opciones de respuesta pictográficas que van de nunca a siempre.

\section{Procedimiento}

Inicialmente se contactaron a los directivos de las escuelas para explicar el propósito del estudio y para obtener la autorización de aplicación del instrumento. El acceso a las primarias fue facilitado por una maestra de la Unidad de Servicios de Apoyo a la Educación Regular (USAER) del Estado de México y dos académicas del posgrado en psicología de la UNAM. Posteriormente, se aplicó la escala a los alumnos de manera grupal en horario acordado con las autoridades educativas. Se agradeció la participación y como retribución se realizó una plática con los padres de familia de las escuelas participantes. Se consideraron las mismas características del estudio y los mismos factores éticos descritos en el procedimiento de la primera fase. Es decir, los padres de familia firmaron el consentimiento informado y la participación fue voluntaria, anónima y confidencial, además de que se obtuvo el asentimiento de los estudiantes.

\section{Análisis de Datos}

Se efectuó un Análisis Factorial Confirmatorio (AFC) a través del programa AMOS (v. 24) con el método de máxima verosimilitud. Finalmente, se calculó el alfa de Cronbach para obtener la confiabilidad de los factores y la escala completa.

\section{Resultados Fase 2}

El modelo presentó los siguientes índices de ajuste: $X^{2}=88.60, g l=53, X^{2}$ $\mid g l=1.67, p<.01 ; C F I=.95, I F I=.95, G F I=.94, A G F I=.92, N F I=.88$, $R M R=.05$ y $R M S E A=.05$. Con base en la literatura en psicometría, se consideran adecuados los siguientes valores: $X^{2}$ normalizado menor a 3 (Martínez et al., 2014), CFI e IFI iguales o mayores a .95 (Furr \& Bacharach, 2008); GFI superior a 90 (Pérez-Gil et al., 2000), $A G F I$ por encima de .90 (Fernández, 2008); y, RMSEA entre .05 y .08 (Martínez et al., 2014). Por lo tanto, los datos muestran evidencia de validez de constructo en la Escala de Autoconcepto Académico para Alumnos de Primaria (EAAAP). Asimismo, el modelo fue parsimonioso, obtenido sin cambiar la escala identificada en la primera fase y sin covarianzas entre errores. En la figura 1 se muestra la representación gráfica del modelo. 
Figura 1

\section{Modelo estructural de las correlaciones de los factores del autoconcepto académico}

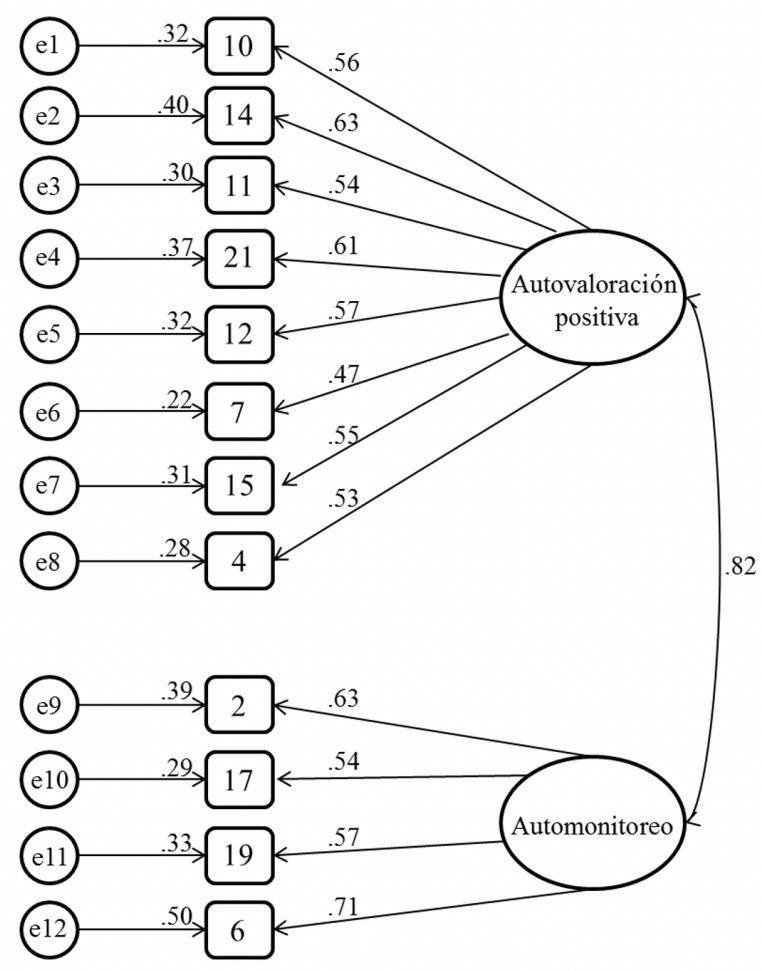

Figura 1

Modelo estructural de las correlaciones de los factores del autoconcepto académico

Con la muestra de esta segunda fase, la confiabilidad de la escala total se mantuvo igual que en la fase inicial, sin embargo, los datos de los factores tuvieron ligeros cambios. En este caso, se encontró un alfa de Cronbach de .84 para el instrumento completo, de .78 en el primer factor (autovaloración positiva) y de .70 en el segundo factor (automonitoreo), los cuales se consideran adecuados con base en Argibay (2006) y Martínez et al. (2014).

\section{Discusión}

Con base en las propiedades psicométricas obtenidas en los resultados, se cumplió el objetivo del estudio al obtener una escala adecuada en la evaluación del autoconcepto académico para alumnos de cuarto a sexto grado de primaria, lo que equivale a niños entre 8 y 12 años de edad. Su relevancia reside en que la EAAAP se enfoca en un momento significativo del desarrollo, lo que posibilita la instrumentación de intervenciones preventivas, en las modificaciones de los ambientes familiar y escolar, y en la instrumentación de programas, talleres y pláticas en la comunidad escolar, para impactar positivamente en la educación de los niños. 
Respecto con las bases teóricas, la escala incluyó ítems de matemáticas y contenidos lingüísticos propuestos en el modelo de dos factores de Byrne y Shavelson (1986) y Marsh (1990), que en este caso particular conformaron los factores de autovaloración positiva y automonitoreo. El primero de éstos fue más congruente con una percepción positiva sobre la capacidad para realizar actividades escolares, tales como; trabajos, exposiciones y resolución de problemas, mientras que el otro se refirió a la percepción vinculada con la capacidad para ejecutar procesos cognitivos, especialmente asociados con las habilidades verbales, como encontrar la idea principal, y repetir y clasificar información.

En comparación con la escala original de Ordaz-Villegas et al. (2013) dirigida a población adolescente, se encontraron las siguientes diferencias. El número de reactivos y factores disminuyó, el nivel de confiabilidad aumentó ligeramente y se obtuvieron más evidencias de validez, aunque la varianza explicada se redujo en $6.72 \%$. Algunas de las discrepancias entre ambos instrumentos pueden ser explicadas con base en las diferencias inherentes entre niños y adolescentes, y a las divergencias en los niveles educativos. Por su parte, la similitud más sobresaliente fue que los factores originales de autorregulación y aptitudes intelectuales de la AAPA (Ordaz-Villegas et al., 2013) correspondieron de manera respectiva, con los de autovaloración positiva y automonitoreo obtenidos en la escala del presente estudio.

Respecto con las características psicométricas, la EAAAP mostró valores óptimos en todas las áreas de la validez, a diferencia de las escalas encontradas. En este sentido, todos los instrumentos encontrados carecen de validez de contenido, y algunas otras, también de criterio, como las construidas por Carranza y Bermúdez-Jaimes (2017); Chávez (2014); Gálvez-Nieto et al. (2017); y Merino y Díaz (2003). El binomio de análisis factorial exploratorio y confirmatorio también se encontró en las pruebas de Carranza y Bermúdez-Jaimes (2017); Gálvez-Nieto et al. (2017); Merino y Díaz (2003), con valores de alfa e índices de ajuste similares a los encontrados en el presente estudio. Sin embargo, se encontró que la EAAAP obtuvo la menor varianza explicada, probablemente debido a que cuenta con menos reactivos y factores.

Cabe destacar que las diferencias de la EAAAP con los instrumentos antes descritos, pueden deberse a las divergencias en las muestras y a las perspectivas teóricas de las que subyacen los reactivos. Por un lado, evaluar el autoconcepto global desde la perspectiva jerárquica de Shavelson et al. (1976) o centrarse en la faceta académica del mismo, y por otro, considerar a éste último como estructurado por autoconceptos específicos de las materias escolares o desde el modelo de dos factores de Byrne y Shavelson (1986) y Marsh (1990).

En cuanto a las limitaciones, se reconoce la falta de evidencia para utilizar el instrumento en muestras diferentes, como estudiantes de los primeros años de primaria y de comunidades rurales. Por otro lado, el dominio de matemáticas como elemento esencial en la estructura del autoconcepto académico (Esnaola et al., 2008; Moreno, 2005), está poco representado en la escala obtenida. Además, los datos psicométricos son 
insuficientes para establecer puntos de corte y puntuaciones normativas. Por lo tanto, se recomienda analizar la inclusión de reactivos sobre el dominio de matemáticas, así como realizar estudios instrumentales adicionales para examinar las propiedades psicométricas en muestras grandes y diversas.

\section{Conclusiones}

El instrumento es capaz de obtener puntuaciones susceptibles a interpretaciones apropiadas sobre el autoconcepto académico en alumnos de educación primaria de la Zona Metropolitana del Valle de México. No obstante, es apropiada para evaluar dicha variable en poblaciones similares, aunque para ello se recomienda analizar sus características psicométricas en diversas muestras. Asimismo, se aconseja realizar adaptaciones para su empleo en diferentes países. Su aplicación es sencilla, rápida y útil en la detección de factores de riesgo y protección relacionados con el proceso educativo de los estudiantes. Puede ser utilizada en el ámbito de la psicología y la educación, como apoyo para instrumentar programas de prevención y en otras investigaciones relacionadas con el tema.

\section{Agradecimientos}

El estudio fue financiado por el Consejo Nacional de Ciencia y Tecnología (beca 449441 y becario 299051) y el Programa de Apoyo a Proyectos de Investigación e Innovación Tecnológica (IN307419).

\section{Referencias}

Álvarez, A., Suárez, N., Tuero, E., Núñez, J., Valle, A., \& Regueiro, B. (2015). Implicación familiar, autoconcepto del adolescente y rendimiento académico. European Journal of Investigation in Health, Psychology and Education, 5(3), 293-311. https://doi.org/10.30552/ejihpe.v5i3.133

American Educational Research Association, American Psychological Association \& National Council on Measurement in Education (2014). Standards for Educational and Psychological Testing. American Educational Research Association.

Argibay, J. C. (2006). Técnicas psicométricas. Cuestiones de validez y confiabilidad. Subjetividad y Procesos Cognitivos, (8), 15-33.

Bustos, V., Oliver, A., \& Galiana, L. (2015). Validación del autoconcepto forma 5 en universitarios peruanos: una herramienta para la psicología positiva. Psychology/Psicología Reflexão e Crítica, 4, 690-697. http://dx.doi.org/10 $.1590 / 1678-7153.201528406$

Byrne, B. M., \& Shavelson, R. J. (1986). On the structure of adolescent selfconcept. Journal of Educational Psychology, 78(6), 474-481. https://doi.o $\mathrm{rg} / 10.1037 / 0022-0663.78 .6 .474$ 
Carranza, R., \& Bermúdez-Jaimes, M. (2017). Análisis psicométrico de la escala de autoconcepto AF5 de García y Musito en estudiantes universitarios de Tarapoto (Perú). Interdisciplinaria, 34(2), 459-472.

Cazalla-Luna, N., \& Molero, D. (2013). Revisión teórica sobre el autoconcepto y su importancia en la adolescencia. Revista Electrónica de Investigación y Docencia (REID), (10), 43-64.

Chávez, B. (2014). Evaluación multidimensional de alumnos con aptitud sobresaliente de educación primaria (Tesis de doctorado). Universidad Nacional Autónoma de México, México.

Creswell, J. (2015). Educational research: Planning conducting, and evaluating quantitative and qualitative research. Pearson.

Durán, T., \& Acle, G. (2019). Escala de motivación escolar para alumnos de primaria: evidencias de su validez y confiabilidad. Manuscrito presentado para su publicación.

Escobar-Pérez, J., \& Cuervo-Martínez, A. (2008). Validez de contenido y juicio de expertos: una aproximación a su utilización. Avances en Medición, 6 , 27-36.

Esnaola, I., Goñi, A., \& Madariaga, J. M. (2008). El autoconcepto: perspectivas de investigación. Revista de Psicodidáctica, 13(1), 69-96.

Fernández-Zabala, A., Goñi, E., Rodríguez-Fernández, A., \& Goñi, A. (2015). Un nuevo cuestionario en castellano con escalas de las dimensiones del autoconcepto. Revista Mexicana de Psicología, 2(32), 149-159.

Fernández, R. (2008). Modelos de medida y análisis factorial confirmatorio. En M. A. Verdugo, M. Crespo, M. Badía \& B. Arias (Eds.), Metodología en la investigación sobre discapacidad. Introducción al uso de las ecuaciones estructurales (pp. 29-41). Publicaciones del INICO.

Ferrando, P. J., \& Lorenzo-Seva, U. (2014). El análisis factorial exploratorio de los ítems: algunas consideraciones adicionales. Anales de Psicología, 30(3), 1170-1175. http://dx.doi.org/10.6018/analesps.30.3.199991

Furr, R. M., \& Bacharach, V. R. (2008). Psychometrics: An introduction. Sage Publications.

Gálvez-Nieto, J. L., Polanco, K., \& Salvo, S. (2017). Propiedades psicométricas de la Escala de Autoconcepto Académico (EAA) en Estudiantes Chilenos. Revista Iberoamericana de Diagnóstico y Evaluación - e Avaliação Psicológica, 1(43), 5-16. https://doi.org/10.24265/liberabit.2018.v24n1. 09

García, F., \& Musitu, G. (2014). AF-5. Autoconcepto Forma 5. TEA Ediciones.

García, R. (2003). Autoconcepto académico y percepción familiar. Revista Galego-Portuguesa de Psicoloxía e Educación, 8(7), 1138-1663.

Gargallo, B., Garfella, P. R., Sánchez, F., Ros, C., \& Serra, B. (2009). La influencia del autoconcepto en el rendimiento académico en estudiantes universitarios. Revista Española de Orientación y Psicopedagogía, 20(1), 16-28.

González-Pineda, J. A., Núñez, J. C., González-Pumariega, S., Álvarez, L., Roces, C., García, M., González, P., Cabanach, R. G., \& Valle, A. (2000). Autoconcepto, proceso de atribución causal y metas académicas en niños con y sin dificultades de aprendizaje. Psicothema, 12(4), 548-556.

González, A. (2005). Motivación académica. Ediciones Pirámide. 
Guay, F., Chanal, J., Ratelle, C., Marsh, H., Larose, S., \& Boivin, M. (2010). Intrinsic, identified, and controlled types of motivation for school subjects in young elementary school children. British Journal of Education Psychology, 80(4),711-735. https://doi.org/10.1348/000709910X49908 4

Ibarra, E., \& Jacobo, H. M. (2016). La evolución del autoconcepto académico en adolescentes. Revista Mexicana de Investigación Educativa (RMIE), 21(68), 45-70.

Jackson, P. (1991). La vida en las aulas. Morata.

Kerlinger, F., \& Lee, H. B. (2002). Investigación del comportamiento. Métodos de investigación en ciencias sociales. McGraw-Hill.

Lloret-Segura, S., Ferreres-Traver, A., Hernández-Baeza, A., \& Tomás-Marco, I. (2014). El análisis factorial exploratorio de los ítems: una guía práctica, revisada y actualizada. Anales de Psicología, 30(3), 1151-1169. http://dx.d oi.org/10.6018/analesps.30.3.199361

Macionis, J. J., \& Plummer, K. (2007). Sociología. Pearson Educación.

Marsh, H. W. (1990). The structure of academic self-concept: The Marsh/ Shavelson model. Journal of Educational Psychology, 82(4), 623-636. http s://doi.org/10.1037/0022-0663.82.4.623

Marsh, H. W., \& Seaton, M. (2013). Academic self-concept. En J. Hattie \& E. M. Anderman (Eds.), International guide to student achievement (pp. 62-63). Routledge.

Martínez, M. R., Hernández, M. V., \& Hernández, M. J. (2014). Psicometría. Alianza Editorial.

Merino, C., \& Díaz, M. (2003). Validez de constructo y confiabilidad de la escala de autoconcepto sobre las habilidades de M. C. Dayton. Revista de Investigación en Psicologia, 6(2), 102-110. https://doi.org/10.15381/rinv p.v6i2.5161

Montealegre, R., \& Forero, L. A. (2006). Desarrollo de la lectoescritura: adquisición y dominio. Acta Colombiana de Psicologia, 9(1), 25-40.

Moreno, G. (2005). Relaciones entre autoconcepto académico, atribuciones de éxito y fracaso, y rendimiento académico en escolares preadolescentes. Revista de Psicología de la PUCP, 33(1), 5-38.

Ordaz-Villegas, G., Acle-Tomasini, G., \& Reyes-Lagunes, L. I. (2013). Development of an Academic SelfConcept for Adolescents (ASCA) scale. Journal of Behavior, Health \& Social Issues, 5(2), 117-130. https://doi.or $\mathrm{g} / 10.5460 /$ jbhsi.v5.2.42304

Orgilés, M., Johnson, B. T., Huedo-Medina, T. B., \& Espada, J. P. (2012). Autoconcepto y ansiedad social como variables predictoras del rendimiento académico de los adolescentes españoles con padres divorciados. Electronic Journal of Research in Educational Psychology, $10(1), 57-72$.

Peralta, F. J., \& Sánchez, M. D. (2003). Relaciones entre el autoconcepto y el rendimiento académico, en alumnos de educación primaria. Revista Electrónica de Investigación Psicoeducativa y Psicopedagógica, 1(1), 95-120.

Pérez-Gil, J. A., Chacón, S., \& Moreno, R. (2000). Validez de constructo: el uso de análisis factorial exploratorio-confirmatorio para obtener evidencias de validez. Psicothema, 12(Su2), 442-446. 
Reyes-Lagunes, I., \& García y Barragán, L. (2008). Procedimiento de validación psicométrica culturalmente relevante: un ejemplo. La Psicología Social en México, 12, 625-636.

Salum-Fares, A., \& Reséndiz-Balderas, E. (2015). Efectividad de un programa de intervención educativa para la mejora del autoconcepto en el ámbito escolar. Revista Mexicana de Psicología Educativa RMPE, 3(1), 23-37.

Salum-Fares, A., Marín, R., \& Reyes, C. (2011). Relevancia de las dimensiones del autoconcepto en estudiantes de escuelas secundarias de Ciudad Victoria, Tamaulipas, México. Revista Electrónica de Psicología Iztacala, 14(2), 255-272.

Santana, L. E., Feliciano, L. A., \& Jiménez, A. B. (2009). Autoconcepto académico y toma de decisiones en el alumno de bachillerato. REOPRevista Española de Orientación y Psicopedagogia, 20(1), 61-75. https://d oi.org/10.5944/reop.vol.20.num.1.2009.11440

Santos, M. V., \& Santos, M. Á. (2013). Estilos de aprendizaje y autoconcepto académico en los alumnos de bachillerato: diferencias entre modalidades. Revista de Estilos de Aprendizaje, 6(11), 100-117.

Schmidt, V., Messoulam, N., \& Molina, F. (2008). Autoconcepto académico en adolescentes de escuelas medias: presentación de un instrumento para su evaluación. Revista Iberoamericana de Diagnóstico y Evaluación - $e$ Avaliação Psicológica, 1(25), 81-106.

Schunk, D. H., \& Pajares, F. (2009). Self-efficacy theory. En K. R. Wentzel \& A. Wigfield (Eds.), Handbook of motivation at school (pp. 35-53). Routledge.

Shavelson, R. J., Hubner, J. J., \& Stanton, G. C. (1976). Self-concept: Validation of construct interpretations. Review of Educational Research, 46(3), 407-441. https://doi.org/10.3102/00346543046003407

Stegers-Jager, K., Cohen-Schotanus, J., \& Themmen, A. (2016). Motivation, learning strategies, participation and medical school performance. Medical Education, 46, 678-688. https://doi.org/10.1111/j.1365-2923.2012.042 84.x

Tristán-López, A. (2008). Modificación al modelo de Lawshe para el dictamen cuantitativo de la validez de contenido de un instrumento objetivo. Avances en Medición, 6, 37-48.

Wang, M-T., \& Eccles, J. (2013). School context, achievement motivation, and academic engagement: A longitudinal study of school engagement using a multidimensional perspective. Learning and Instruction, 28, 12-23. https ://doi.org/10.1016/j.learninstruc.2013.04.002 